\title{
Intelligent Event Management with Bluetooth Sensor Networks
}

\author{
Versichele, M., Huybrechts, R., Neutens, T., Van de Weghe, N. \\ Department of Geography \\ Ghent University \\ Ghent, Belgium \\ mathias.versichele@ugent.be
}

\begin{abstract}
This paper outlines the potential use of Bluetooth tracking of mobile devices in an urban environment for an intelligent and adaptive management of large-scale events. The used methodology is explained in detail, and its information potential is demonstrated through results that were gathered and processed offline. Additionally, a near-future project where the methodology will also generate real-time information is outlined, together with the system architecture necessary for this aim.
\end{abstract}

Keywords: Intelligent Event Management; Bluetooth Tracking; Bluetooth Sensor Network; Real-Time Information Processing.

\section{INTRODUCTION}

In recent years, several technological advances and the increasing penetration of ever more intelligent mobile devices have caused a rapid increase in the availability of contextual data concerning our everyday lives. Social and community intelligence (SCI) research aims to discover interesting patterns and knowledge from communities by mining these digital traces [1]. One specific type of information is location, and a large focus in SCI is indeed reserved for studying human mobility. Several methodologies and technologies have been used in order to extract mobility information from mobile devices ranging from mining data logs of mobile phone operators [2], [3], to the use of the global positioning system (GPS) technology [4]. Less evident, Bluetooth has also recently been proposed as a valuable alternative tracking technology in different domains such as urban design [5] and social studies [6].

While this technology - together with others - could make environments more intelligent and adaptive to its inhabitants in a large number of ways [7], we wish to focus this paper on the intelligent management of (large-scale) events. Bluetooth tracking has already been used to study the spatiotemporal dynamics of crowds in a variety of settings, such as during the Ghent Festivities 2010 where around 150.000 trajectories over 10 days were generated, accounting for around $10 \%$ of the total visitor population being tracked [8]. In the near future the methodology will be used to generate and disseminate realtime information during the Ghent Light Festival 2012. This information will be used to generate a number of basic location based services including the display of average queuing times for certain attractions.
The paper will start by giving more details about the working principle of the Bluetooth tracking methodology and our implementation in section II. Next, in section III, we highlight the information potential residing in the resulting datasets by focusing on a selection of possibilities and highlighting results from the Ghent Festivities 2011 project. In section IV, we focus on the real-time information processing and dissemination by outlining our aims for the Ghent Light Festival 2012 and describing the system architecture that will support these processes.

\section{Bluetooth Tracking Methodology}

\section{A. Working Principle}

Bluetooth is a short-range, low-power and open protocol for implementing Wireless Personal Area Networks (WPAN) between mobile devices. In brief, the establishment of a connection between two devices consists of a discovery phase followed by a connection phase. In the discovery phase, a master device enters the inquiry sub-state and starts transmitting inquiry packets, which triggers other devices within the detection range of the master device that are in the inquiry scan sub-state (corresponding to 'discoverable' devices) to respond by transmitting an identification message.

The Bluetooth tracking methodology uses this discovery protocol to generate spatiotemporal information from the movements of Bluetooth devices carried around by their owners. A Bluetooth scanner (depicted in Figure 1) can sense the presence of discoverable Bluetooth devices in its vicinity by continuously repeating the previously mentioned inquiry phase and logging the broadcast messages sent by responding mobile devices within the scanners communication range. Every time a device is detected, its MAC address (unique identifier of the device), COD code (indicating whether it is a phone, smartphone, car kit, etc.), the timestamp of the detection, and the received signal strength indicator (RSSI) of the inquiry response are logged. Because some users include personal information in the friendly name of the detected device (name, phone number, etc.), it is not registered to safeguard privacy. The communication of this information does not require an active connection between the scanner and the mobile device, so the methodology does not need an active cooperation of the tracked individual. 


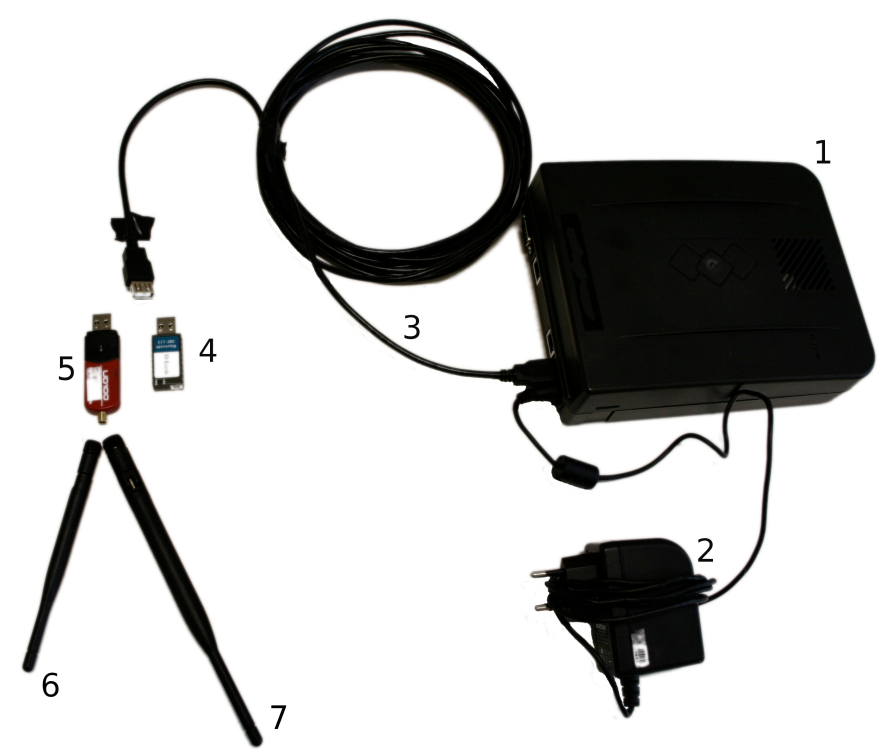

Figure 1. Components of a Bluetooth scanner for tracking purposes: computational unit (1), power source (2), USB cable (3), class 2 Bluetooth dongle with internal antenna (4), class 1 Bluetooth dongle (5), and $3 \mathrm{dBi}(6)$ and $5 \mathrm{dBi}(7)$ omnidirectional antennas.

By placing Bluetooth scanners at different strategic locations, meaningful trajectories generated by mobile devices (and correspondingly by their owners) can be reconstructed. Because of the complex environmental settings and the resulting unpredictability of the propagation of Bluetooth signals, positioning is currently done through the proximity principle, where the position of a detected mobile device is approximated to the point-position of the sensor by which it is detected. The movements of mobile devices that are not within range of any scanner cannot be reconstructed.

The spatial granularity of the resulting trajectories ultimately depends on the detection range of the Bluetooth sensors, and on the number and coverage of Bluetooth scanners within the study area. In theory, this detection range depends on the power class of the Bluetooth sensor (class 1: $100 \mathrm{~m}$, class 2: $10 \mathrm{~m}$, class 3: $1 \mathrm{~m}$ ). In practice, however, this range is equally dependent on the environment affecting the travel of signals between the scanner and the detected mobile device. Obstructions like buildings, furniture and other people will lower the detection range at certain angles substantially by preventing line of sight (LOS) communication. Because some of these factors are continuously changing, the exact detection range is not predictable.

\section{B. Scanning Hardware and Software}

Figure 1 shows the hardware components used in the Bluetooth tracking methodology. A Bluetooth scanner is actually a combination of a computational unit running the scanning software and processing and storing the results (1), a power source (2), and a USB cable (3) to connect the computational unit with a Bluetooth sensor (4-5). The heart of the computational unit is an ALIX motherboard (alix $2 \mathrm{~d} 2$, alix $3 \mathrm{~d} 2$, alix6f2), equipped with a 1GB CompactFlash card for storing $\log$ files. The operating system is an adapted version of Voyage Linux, and the scanning software, Gyrid
(http:/github.com/Rulus/Gyrid), is developed at our research group. It is a Python implementation built around the BlueZ Bluetooth stack.

In order to control the detection range, we employ Bluetooth sensors with different detection ranges (class 2: DLink DBT-122 and others; class 1: Sena Parani UD-100). These are respectively shown as numbers 4 and 5 in Figure 1. At most locations, class 2 devices without an external antenna are used. Where a larger detection range is necessary, a class 1 device with a replaceable antenna is used. Two types of omnidirectional antenna are available with gains of 3 (6) and 5 $\mathrm{dB}_{\mathrm{i}}$ (7). The higher the gain of the antenna, the larger the detection range.

\section{INFORMATION POTENTIAL: GHENT FESTIVITIES 2011}

In this section we wish to highlight the current information potential of our methodology. As we already mentioned, the ultimate aim of our methodology is real-time information delivery. Since the real-time capabilities were still partly under development at the moment of writing, we will show results that were generated offline in a previous project: the Ghent Festivities 2011 [9]. Analogous output will, however, be generated in real-time in another project in the near future. More information on that project, our aims and our implementation are given in section IV.

In theory, there are almost no limits to the types and amount of information that can be generated from our mobility datasets. As a first step, we will limit ourselves to three types of information that are relatively easy to extract and disseminate to both event organizers and visitors. These three types of information are essentially linked to the presence of a device $d$ at a scanner location $l\left(P_{d, l}\right)$, the travel of a device $d$ between two scanners at locations $l$ and $m\left(T_{d, l \rightarrow m}\right)$, and the $d$ welling of a device $d$ at a scanner location $l\left(D_{d, l}\right)$. Extrapolating to the entire population of detected devices or 'crowd' (c), these three concepts are translated into the concepts of crowdedness at a location $l\left(C_{l}\right)$, a flow between locations $l$ and $m\left(F_{l \rightarrow m}\right)$ and crowd dwelling at a location $\left(D_{c, l}\right)$. Each of these three concepts will now be discussed in more detail.

The crowdedness $C_{l, T}$ at location $l$ can now be defined as the number of detected devices registered by the scanner at location $l$ during time period $T$. Three important remarks should be made. First, this number only counts devices and not persons. In order to extrapolate to an entire population of visitors, the detection ratio should be investigated. From previous projects we know that this rate can depend on the type of venue and type of visitors being tracked, but generally speaking we track around $10 \%$ of a general public. Second, this number does not take into account the area covered by the Bluetooth sensor and consequently it should not be interpreted as a crowd density. Third, the interpretation also depends on the length of the time period. If this time period is rather small (e.g. one minute) it can almost be considered as a nearimmediate crowdedness as sensed by the public on site, if it is longer (e.g. one hour) it rather indicates the throughput of people that have passed the location during that time period. As an example, Figure 2 shows the temporal variation of the (immediate) crowdedness at a public square during one day of 
the Ghent Festivities 2011. The graph shows a very detailed image of the crowdedness building up to a peak moment around 7 PM, subsiding to a lower level between 9 and 10 PM, and rising to a (lower) peak level again around 11:30 PM. It is also very interesting to look at the link between artist performances on that public square and the crowdedness. Especially the effect of the fourth performance is noteworthy as it coincides with the highest crowdedness of the day. At that moment, the local football team was presented to the football fans. The secondary axis shows the estimated crowd density taking into account the area covered by the sensor. Although these values should be regarded as rough estimates, the density seems to stay well below the critical value of 4 persons $/ \mathrm{m}^{2}$ as stated in [10], and below the value of 2 persons $/ \mathrm{m}^{2}$ used by the event organizers. Figure 3 shows the spatial variation of crowdedness over the entire event area during a one-hour timewindow. It is important to stress that while informative, this visualization should be interpreted with care. Only the values that are located at the sampling points (in our case the locations of the scanners) are real, the values at all other locations are interpolated. As such, the crowdedness estimates at locations that are further away from locations covered by Bluetooth scanners are less reliable. As far as crowdedness figures such as these go, one should always take into account the inherent uncertainties of the methodology: the approximated detection area around a sensor and the uncertain detection ratio. Regardless however, more general trends and patterns - both in time and space - can be extracted and interpreted.

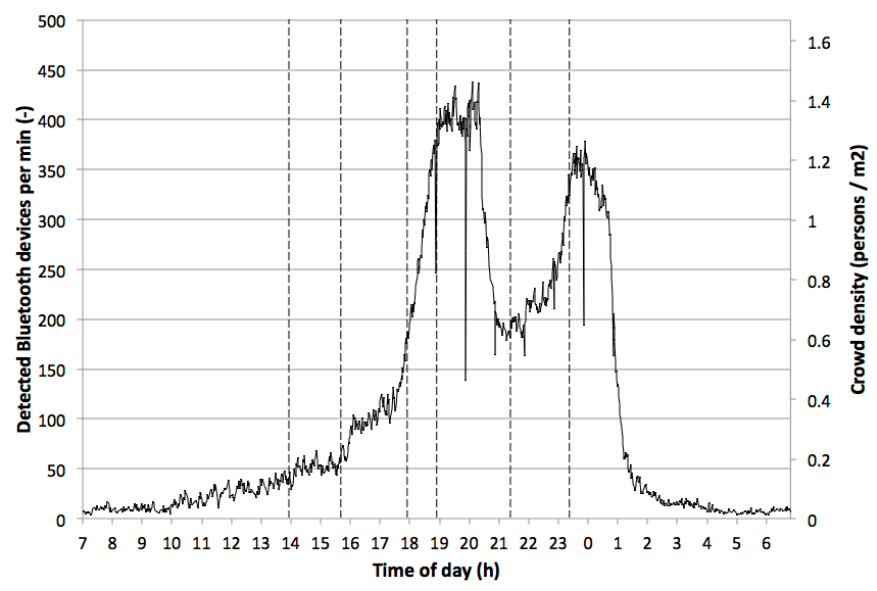

Figure 2. Temporal variation of (immediate) crowdedness at location 1 (see Figure 3) depicted with a time resolution of one minute. The temporal extent encompasses the third day of the Ghent Festivities 2011 festival $(18 / 07 / 2011 \rightarrow 19 / 07 / 2011)$. Vertical dashed lines correspond to beginning times of artist performances at the location.

As previously stated, a flow consists of a set of travels performed by detected devices. A flow can be characterized by two indicators: its size and its average travel speed. The size of a flow, $\operatorname{size}\left(F_{l \rightarrow m, T}\right)$, is defined as the number of detected devices that perform the travel during the time period $T$. A

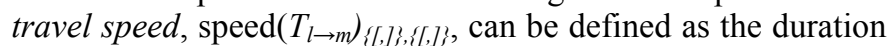
of the time interval between a detection by the sensor at location 1 and a detection by the sensor at location $m$ divided by the distance between both locations, where both detections can either be the first detection at that location (' $[$ '), or the last detection (']'). This leads to 4 variations of travel speeds: $\operatorname{speed}\left(T_{l \rightarrow m}\right)_{[,]}, \operatorname{speed}\left(T_{l \rightarrow m}\right)_{],[}, \operatorname{speed}\left(T_{l \rightarrow m}\right)_{[,[}, \operatorname{speed}\left(T_{l \rightarrow m}\right)_{],]}$. The appropriate choice of both detections depends on the context. In Figure 4, for example, we chose the last detection at the first visited location and the first detection at the second visited location ('][') to characterize the bidirectional flow between locations 7 and 18. The temporal variation of the travel speeds shows a clear drop in the average travel speed in both directions between 1 and 3 PM.

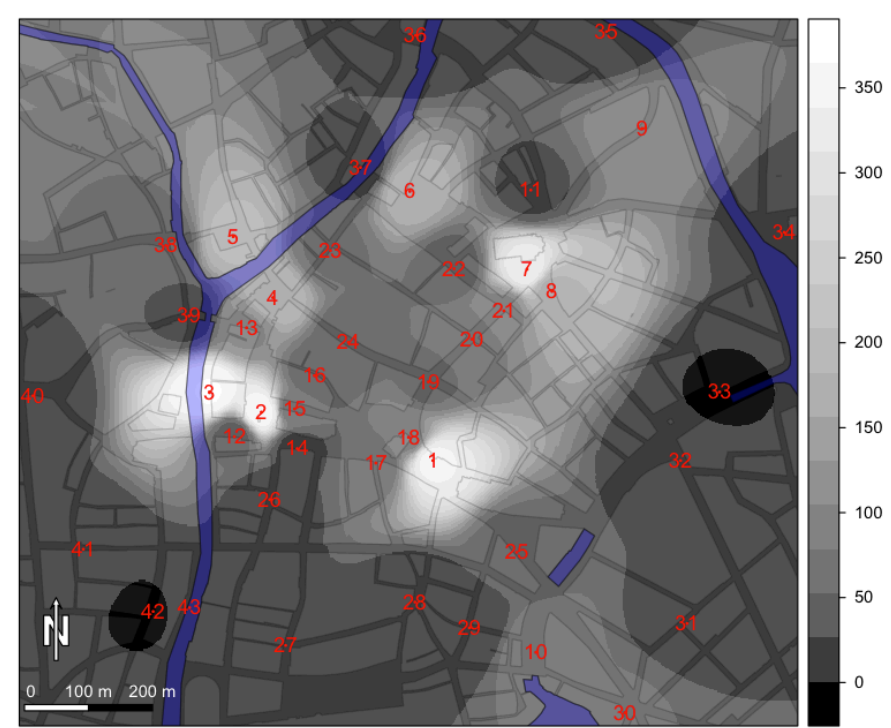

Figure 3. Spatial variation of crowdedness over the city center of Ghent, Belgium during the Ghent Festivities 2011. Color values represent the crowdedness levels (during a 5-minute time window starting at 21/07/2011 21:00:00) over the study area (interpolation in $\mathrm{R}$, type=inverse distance weighted, $n=10$, $i d p=5$ ). The labels indicate the locations of the Bluetooth scanners used in the study. Waterways are drawn in blue, buildings in transparent grey.

The dwelling of a device $d$ at location $l, D_{d, l}$, can be defined as the time duration between the first detection of the device at the location and the latest detection of the device at the same location. Additionally, the dwelling is characterized by a property indicating whether the last detection of the device at that location is also the last detection of the device in general or not, corresponding, respectively, to a device which is (as far as the system knows) still located at the location $\left(D_{d, l} *\right)$, or to a device which is known to have had a dwelling time of a certain duration at the location but has since moved to a different location $\left(D_{d, l}\right)$. Extrapolating to the entire population, the crowd dwelling at a location $D_{c, l}$ is composed of all individual dwellings at the location and characterized by an appropriate average value.

\section{REAL-TIME INFORMATION POTENTIAL: GHENT Light FESTIVAL 2012}

In this section, we will focus on real-time information delivery, the system architecture necessary for supporting this and the application in a near-future project. 


\section{A. System Architecture}

The architecture of our system consists of three levels: the clients, the middleware server and the database server. The clients are the Bluetooth scanners which act as nodes in the Bluetooth Sensor Network. Each scanner functions as a gateway that connects immediately to the middleware on the server machine, either over $3 \mathrm{G}$ or over a wired connection depending on the connection possibilities at the scanners location. The client communicates with the middleware through a TCP socket, encrypted with SSL. The middleware was developed in Python and acts as the connection between the clients and the database server. Additionally, it serves as a status dashboard (Figure 5). The database server was developed in Java, and uses $\mathrm{db} 4 \mathrm{o}$ as the database implementation, which is an open-source object-oriented database. The communication between the middleware and the database server happens over a local TCP connection. All queries over the database also run over TCP sockets communicating directly with the database server.
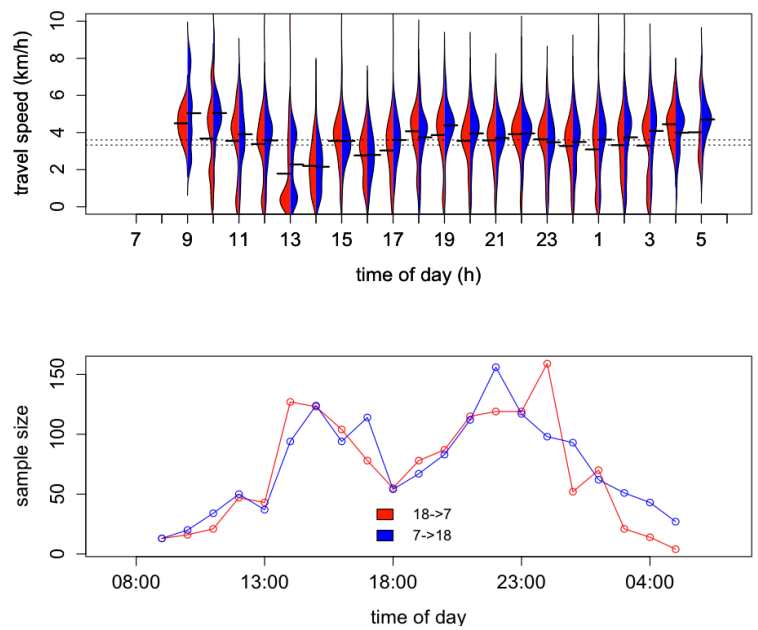

Figure 4. Top: temporal variation (resolution of one hour) of the travel speeds associated with the flow from location 18 to $7\left(\operatorname{speed}\left(\mathrm{T}_{18 \rightarrow 7}\right)_{],}\right.$, , drawn in red), and the flow from location 7 to $18\left(\operatorname{speed}\left(\mathrm{T}_{7 \rightarrow 18}\right)_{],[}\right.$, drawn in blue). The beanplot shows the distribution of travel speeds in both directions. The solid horizontal lines at each side of each beanplot depict the mean value of that distribution. Bottom: temporal variation (resolution of one hour) of the size of the flow from location 18 to 7 (drawn in red) and from location 7 to 18 (drawn in blue). In both cases, the temporal extent encompasses 24 hours ranging from 16/07/2011 07:00:00 until 17/07/2011 07:00:00.

\section{B. Future Project: Ghent Light Festival 2012}

The Ghent Light Festival [11] is a festival highlighting the use of different forms of light in creative, new and unexpected ways. During 4 days, visitors can - free of charge - follow a 4kilometer path taking them to 29 exhibits dispersed over the ancient city center of Ghent, Belgium. During its first edition in 2011, the event drew over 200.000 visitors over 3 days causing unexpected challenges for the organizers. In order to cope more rationally with these challenges in the future, and due to our previous experiences with large events organized in Ghent [8], a cooperation was set up with our research group.

The aim of the project is to disseminate real-time information to both event organizers as well as visitors. As a first step, the real-time information will be limited to the three indicators discussed in section III: crowdedness, travel time, and dwelling. Crowdedness maps like the one in Figure 3 will be shown to the public. The travel time concept will be used to calculate queuing times, as well as the dwelling time inside buildings or on squares.

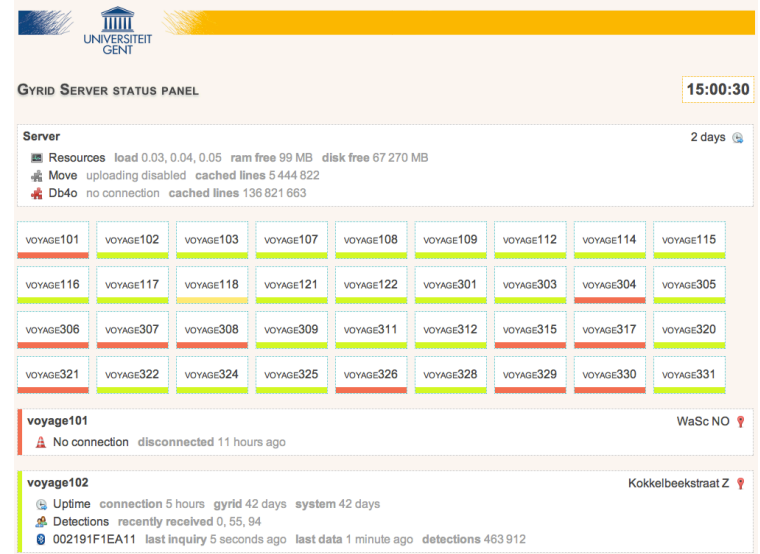

Figure 5. Graphical user interface of the middleware component acting as a dashboard signaling technical problems in real-time.

\section{ACKNOWLEDGMENTS}

We wish to thank the Agency for Innovation by Science and Technology in Flanders (IWT) to fund this research, as well as the City Department of Ghent and all other stakeholders of the Ghent Festivities \& Ghent Light Festival for their input and cooperation.

\section{References}

[8] M. Versichele, T. Neutens, M. Delafontaine, and N. Van de Weghe, "The use of Bluetooth for analysing spatiotemporal dynamics of human movement at mass events: A case study of the Ghent Festivities," Applied Geography, vol. 32, no. 2, pp. 208-220, Mar. 2012.

[9] "Gentse Feesten 2011," 2011. [Online]. Available: http://www.gentsefeesten.be/.

[10] J. J. Fruin, The causes and prevention of crowd disasters. London: , 1993.

[11] "Lichtfestival Gent 2012," 2012. [Online]. Available: http://www.lichtfestivalgent.be/. 